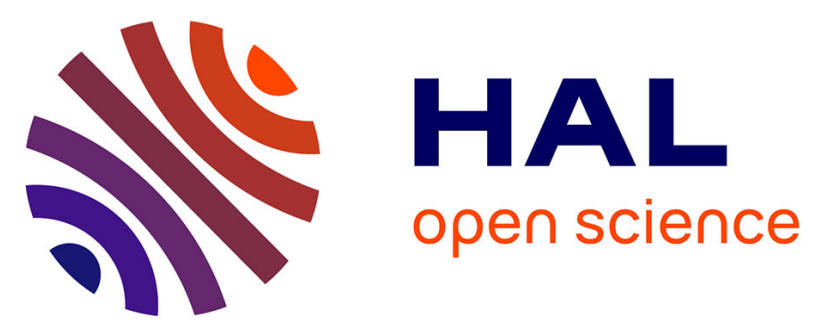

\title{
fMRI-Based Brain Responses to Olfactory Stimulation with Two Putatively Orexigenic Functional Food Ingredients at Two Different Concentrations in the Pig Model
}

Nicolas Coquery, Sophie Menneson, Paul Meurice, Regis Janvier, Pierre

Etienne, Virginie Noirot, David Val-Laillet

\section{To cite this version:}

Nicolas Coquery, Sophie Menneson, Paul Meurice, Regis Janvier, Pierre Etienne, et al.. fMRI-Based Brain Responses to Olfactory Stimulation with Two Putatively Orexigenic Functional Food Ingredients at Two Different Concentrations in the Pig Model. Journal of Food Science, 2019, 84 (9), pp.2666-2673. 10.1111/1750-3841.14772 . hal-02281404

HAL Id: hal-02281404

https://hal-univ-rennes1.archives-ouvertes.fr/hal-02281404

Submitted on 26 Nov 2019

HAL is a multi-disciplinary open access archive for the deposit and dissemination of scientific research documents, whether they are published or not. The documents may come from teaching and research institutions in France or abroad, or from public or private research centers.
L'archive ouverte pluridisciplinaire HAL, est destinée au dépôt et à la diffusion de documents scientifiques de niveau recherche, publiés ou non, émanant des établissements d'enseignement et de recherche français ou étrangers, des laboratoires publics ou privés. 
fMRI-based brain responses to olfactory stimulation with two putatively orexigenic functional food ingredients at two different concentrations in the pig model

Nicolas Coquery ${ }^{1}$, Sophie Menneson ${ }^{1,2}$, Paul Meurice¹, Régis Janvier¹, Pierre Etienne², Virginie Noirot ${ }^{2}$, David Val-Laillet ${ }^{1}$

${ }^{1}$ INRA, INSERM, Univ Rennes, Nutrition Metabolisms and Cancer, NuMeCan, Rennes, France

${ }^{2}$ Phodé, Terssac, France

\section{Corresponding author:}

Nicolas Coquery, INRA UMR1341 NuMeCan, 35590 St Gilles, France nicolas.coquery@inra.fr

Running title: fMRI responses to olfactory stimulation in pig 


\begin{abstract}
Natural plant extracts are increasingly used as functional feed ingredients in animal husbandry and food ingredients in human alternative medicine to improve welfare and health. We investigated in 20 growing pigs via fMRI the brain Blood Oxygen Level-Dependent (BOLD) responses to olfactory stimulation with two sensory functional feed ingredients, A and B, at two different concentrations. Functional ingredient A contained extracts from Citrus sinensis (60-80\%), and ingredient B contained a mixture of extracts Oreganum vulgarae (40-55\%) and Cymbopogon flexuosus (20-25\%). Increased concentration of ingredients induced a higher activation in reward and cognitive areas compared to lower concentrations. Moreover, considering both ingredients at the highest concentration, the ingredient A elicited higher brain responses in brain areas involved in hedonism/pleasantness compared to ingredient B, and more specifically in the caudate nucleus and orbitofrontal cortex. Our findings shed new light in the scope of emotion regulation through olfactory modulation via sensory functional ingredients, which opens the way to further preclinical studies in animal models and translational research in the context of nutrition, welfare, and health.
\end{abstract}

\title{
Key words
}

Olfaction; Functional food ingredients; Pig; Brain; fMRI; Hedonism.

\section{Practical Application}

Functional food/feed ingredients are gaining interest for improving health and welfare in humans and animals. Besides representing an alternative to antibiotics for example, food ingredients and their sensory characteristics might have a positive impact on emotions and consequently on well-being. Functional brain imaging in large animals such as in the pig model is a promising approach to investigate the central and behavioural effects of food 
ingredients, and determine the most effective blends and concentrations to modulate internal and emotional states. 


\section{Introduction}

The use of natural plant extracts as functional food ingredients is gaining interest due to both their impacts on welfare and health security (Piątkowska \& Rusiecka-Ziółkowska, 2016), e.g. as an alternative to antibiotics in animal production, and their potential to prevent chronic diseases in humans (van Breda \& de Kok, 2018). Besides an obvious post-ingestive impact on microbiota (Cairo et al., 2018; Dudek-Wicher, Junka, \& Bartoszewicz, 2018), intestinal

physiology (Patra, Amasheh, \& Aschenbach, 2018), hormonal regulation (Bower, Real Hernandez, Berhow, \& de Mejia, 2014), and immunity (Williams et al., 2017), food ingredients from natural plant extracts might also act directly on exteroception, and notably olfaction. Olfactory stimulations can elicit specific brain responses related to emotions and hedonic valuation (Billot et al., 2017; Sorokowska et al., 2017; Soudry, Lemogne, Malinvaud, Consoli, \& Bonfils, 2011).

In a previous series of studies using the pig model, we investigated 8 different sensory functional feed ingredients, which induced different outcomes in terms of feed preferences and eating behavior (Clouard \& Val-Laillet, 2014). Based on these behavioral data, we further investigated the impact of one of these ingredients (mainly composed of Citrus sinensis extracts) on brain glucose metabolism via ${ }^{18} \mathrm{~F}$-fluoro-deoxy-glucose (FDG) positron emission tomography (PET), and in response to olfactogustatory response with or without prior familiarization to the ingredient (Val-Laillet, Meurice, \& Clouard, 2016). Because the FDGPET imaging modality can test only one condition per imaging session, we developed a functional magnetic resonance imaging (fMRI) approach in the pig model to discriminate brain responses to different gustatory stimulations (Coquery et al., 2018). Here, we adapted this set-up to olfactory stimulation to evaluate brain responses to the odor of two feed ingredients at different concentrations within the same imaging session. 
Among natural product-based extracts, fruit extracts, such as Citrus sinensis, present some promising properties for food intake regulation and mood modulation (Dosoky \& Setzer, 2018; Igarashi, Ikei, Song, \& Miyazaki, 2014; Lamport et al., 2016). Herbal extracts also present some interesting features. Oreganum vulgarae and its principal compounds, carvacrol, as well as Cymbopogon flexuosus, i.e. lemongrass, can have positive effects on behavior and emotions (Amiresmaeili, Roohollahi, Mostafavi, \& Askari, 2018; Blanco, Costa, Freire, Santos, \& Costa, 2009; Costa et al., 2011; Jugl-Chizzola et al., 2006; Mechan et al., 2011; Pires et al., 2013; Sharifi-Rad et al., 2018; Zotti et al., 2013). In this study, we compared two food ingredients with putative orexigenic and hedonic outcomes: ingredient A, derived from Citrus sinensis extracts, and ingredient $\mathrm{B}$, a combination of Oreganum vulgarae and Cymbopogon flexuosus extracts. First, given that the hedonic value of an odor can be related to the concentration used (Clouard, Jouhanneau, Meunier-Salaün, Malbert, \& Val-Laillet, 2012), we analyzed the impacts of two different concentrations of each ingredient on brain responses to identify the best concentration for comparing ingredients. We also aimed at identifying the ingredient that promoted the highest outcomes on the reward circuit. Second, we characterized the specific neuronal networks modulated by each ingredient and the associated neurocognitive functions involved based on the scientific literature.

\section{Materials and Methods}

\subsection{Animals}

Experiments were conducted in accordance with the current ethical standards of the European Community (Directive 2010/63/EU), Agreement No. C35-275-32 and Authorization No. 3588. The Regional Ethics Committee in Animal Experiment of Brittany has validated and approved the entire procedure described in this paper (project $\mathrm{N}^{\circ}$ 2017070518585877). A total of twenty eighteen-week-old Piétrain x (Large White/Landrace) female pigs were used in 
this study investigating the brain response to food ingredients. Before imaging, these pigs were used initially in a research project investigating the gut-brain adaptations to a six-week psychosocial stress to describe a new relevant preclinical model, and were separated into two groups. These two groups (10 animals per group) differed only in their housing conditions: either housed alone in individual pens $\left(150 \times 60 \times 80 \mathrm{~cm}^{3}\right)$ without environmental enrichment, or by two animals in twice larger pens $\left(150 \times 120 \times 80 \mathrm{~cm}^{3}\right)$ with environmental enrichments (a balloon and a suspended chain). Animals housed in individual pens were also exposed to unpredictable environmental visual and auditory stimuli (noise and rotating red light warning during day, every ten minutes, and night, every sixty minutes). For the statistical analysis, group attribution was included as a covariable. For all animals, the room was maintained at $\sim 24^{\circ} \mathrm{C}$ with a natural light-dark cycle (from April to June in French Brittany).

\subsection{Anesthesia}

For brain imaging, pre-anesthesia was performed with an intramuscular injection of ketamine (5 mg/kg - Imalgene 1000, Merial, Lyon, France) in overnight-fasted animals, i.e. imaging being performed 24 to 30 hours after the last meal distribution. Isoflurane inhalation (Aerane $100 \mathrm{ml}$, Baxter SAS, Guyancourt, France) was used to suppress the pharyngotracheal reflex and then establish a surgical level of anesthesia, 3-5\% and 2-3\% respectively. After intubation, anesthesia was maintained with $2.5-3 \%$ isoflurane and mechanical ventilation allowed adjustment of respiratory frequency at 17 breaths/minute with a tidal volume of $650 \mathrm{ml}$. Cotton wool with an additional headset was used to conceal the animal's ears and tape was used to keep the eyes closed.

\subsection{Olfactory stimulation}


We used an improved custom-made stimulation apparatus quite similar to that used in previous studies (Clouard, Jouhanneau, Meunier-Salaün, Malbert, \& Val-Laillet, 2012), located outside the magnet-shielded room (5-m distance) to deliver olfactory stimulations. Briefly, animals were equipped with a tube inserted in the right nostril, allowing air circulation in the entire nasal cavity.

Two functional ingredients formulated by Phodé (Terssac, France) were used in this study: the ingredient A was mainly made of a natural extract of orange, Citrus sinensis (60-80\%), and the ingredient $B$ was mainly made of a natural extract of oregano, Origanum vulgare (40$55 \%$ ) and lemongrass, Cymbopogon flexuosus (20-25\%). Vehicle for both solutions was composed of distillated water (60-80\%) and glyceryl polyethylene glycol ricinoleate (2040\%). For olfactory stimulation, solutions A and B were diluted in distillated water (1 L) at two concentrations (A1: $0.0105 \%$; A10: $0.105 \%$; B1: $0.02 \%$; B10: $0.2 \%$ ). The lowest concentration of ingredient A was comparable to the concentration used in a previous pig study (Val-Laillet, Meurice \& Clouard, 2016). The lowest concentration of ingredient B was selected in order to get the same odor intensity (assessed by a human expert panel). The control solution consisted of the vehicle diluted in distilled water at the higher concentration used for ingredients (Control: $0.2 \%$ ). In order to obtain the highest brain responses for each animal, one block of stimulation/acquisition was performed for each ingredient and concentration: ingredient stimulation (30 sec, $4 \mathrm{~L} / \mathrm{min}$ ), and control stimulation (30 sec, $4 \mathrm{~L} / \mathrm{min}$ ) repeated 8 times. The order of ingredients was: $\mathrm{A}$ then $\mathrm{B}$, from the lowest concentration to the highest for each ingredient in order to reduce odor saturation, resulting to the following order: A1, A10, B1, B10. The stimulation protocol duration per ingredient and per concentration was about $4 \mathrm{~min}$, i.e. $16 \mathrm{~min}$ in total for both ingredients at two different concentrations. 


\subsection{MRI image acquisition}

Imaging was performed during five days, with four animals being imaged per day. Image acquisition was performed as previously described (Coquery et al., 2018) on a 1.5-T magnet (Siemens Avanto, Erlangen, Germany) at the Rennes Platform for Multimodal Imaging and Spectroscopy (PRISM). Acquisitions were performed using a combination of coils (Body and Spine matrix coils) for optimized signal to noise ratio acquisition. T1 weighted anatomical image acquisition: a MP-RAGE sequence was adapted for adult minipig anatomy $\left(1.2 \times 1.2 \times 1.2 \mathrm{~mm}^{3}, \mathrm{NA}=2, \mathrm{TR}=2400 \mathrm{~ms}, \mathrm{TE}=3.62 \mathrm{~ms}, \mathrm{TI}=854 \mathrm{~ms}, \mathrm{FA}=8^{\circ}\right.$, acquisition duration $15 \mathrm{~min}$ ). BOLD (Blood-Oxygen-Level Dependent) signal acquisition: an echo planar imaging sequence was adapted for pig head geometry (TR/TE: 2500/40 ms, FA: $90^{\circ}$, voxel size: $2.5 \times 2.5 \times 2.5 \mathrm{~mm}^{3}$ ). The first four acquired volumes were excluded for the data analysis, meaning that no stimulation was performed during this period. For some animals, we detected a loss of MR signal in some part of the frontal lobes due to the anatomical presence of an air cavity anterior to the brain. The part of the frontal lobe that was not covered in all animals was thus excluded from the data analysis and is depicted as a dark grey area in the global brain activation maps (Figures 1-3).

\subsection{Data analysis and statistical image analysis}

Data analysis was performed with SPM12 (version 6906, Wellcome Department of Cognitive Neurology, London, UK). After slice timing correction, realignment and spatial normalization on a pig brain atlas (Saikali et al., 2010), images were smoothed with a Gaussian kernel of $4 \mathrm{~mm}$. Due to limitations related to the size of the pig brain and the effect of anesthesia on brain activity, we used a non-standard statistical analysis with regards to human statistical standards. Further details regarding the validity and limitations of the statistical approach used in this model and paradigm are described in Coquery et al. (2018). Voxel-based statistic: first- 
level (within-individual contrast) and second-level (within-group contrast) statistics were assessed with a threshold set at $\mathrm{p}<0.05$ to produce the brain maps of activation. SVC-based statistics (Small Volume Correction): anatomical regions of interest (ROIs) from the Saikali pig brain atlas (Saikali et al., 2010) were used for SVC-based statistics with a $p$-value corrected for multiple ROIs comparison with a Bonferroni correction and at a threshold of 0.05 (peak level). For the analysis of the brain responses for each ingredient at different concentrations, sixteen ROIs corresponding to eight bilateral brain structures were used based on a priori hypothesis from a previous study performed with similar food ingredients (ValLaillet, Meurice, \& Clouard, 2016), (i) olfactory and gustatory sensory brain regions: prepypriform area and insular cortex; (ii) motivational and reward brain regions: caudate nucleus and putamen; (iii) associative learning brain regions: amygdala and parahippocampal cortex; and (iv) hedonic and executive brain regions: anterior prefrontal cortex and dorsallateral prefrontal cortex. The related uncorrected $p$-value threshold after Bonferroni correction was 0.0031 corresponding to a corrected $p$-value of 0.05 . Uncorrected $p$-values between 0.0031 and 0.005 were considered as a statistical trend. For the comparison between ingredients, the brain regions related to olfaction and gustation were not included, resulting in a number of six analyzed brain regions corresponding to twelve ROIs bilaterally. The related uncorrected $p$-value after Bonferroni correction was 0.0042 corresponding to a corrected $p$ value of 0.05 . Uncorrected $p$-values between 0.0042 and 0.005 were considered as a statistical trend.

For voxel-based statistics and SVC-based statistics, no suprathreshold voxels were detected with FDR (False Discovery Rate) correction at $\mathrm{p}<0.05$. Due to technical problem during functional acquisition, i.e. removal of the stimulation tube or animal heads movement, only sixteen animals were used for analysis. 


\section{Results}

\subsection{Brain responses to the ingredient $\mathrm{A}$}

Brain activation maps (Figure 1A). Independent of the concentration used, ingredient A promoted brain responses in sensory brain regions including the prepyriform area (PP), an olfactory relay/center, and the insular cortex (IC), a main gustatory center, in reward and motivational brain regions including the caudate nucleus $(\mathrm{Cd})$, the putamen (Put) and the nucleus accumbens (Ac), and in associative learning brain regions, including a decreased activity in the amygdala (AMY). A ten-fold increase of ingredient A concentration (A10 vs. A1) promoted modulations of brain activation in sensory brain regions including the $\mathrm{PP}$, in cingulate cortex areas (Ventral-Posterior: VP-CC, Dorsal-Posterior: DP-CC and DorsalAnterior: DA-CC), in the associative learning brain regions including a decreased activation in the AMY and the parahippocampal cortex (PHC). We could also detect an increased activation in reward and motivational brain regions, and in prefrontal brain regions, including the anterior prefrontal cortex (A-PFC) and the orbitofrontal cortex (OFC).

Corrected SVC-based statistic (Figure 1B). We detected an impact of ingredient A1 in reward and motivational brain regions: increased activation in the PUT and decreased activation in the $\mathrm{Cd}$ whereas A10 promoted a modulation in brain responses in the IC and a tendency toward a reduced activation in the A-PFC. A ten-fold increased of the ingredient $\mathrm{A}$ concentration (A10 vs A1) promoted a modulation in the reward and motivational brain regions, including a tendency toward increased activation in the PUT, a decreased activation in the IC and the PHC, and a tendency toward increased activation in the A-PFC. Of interest, is the fact that opposing brain responses were seen in the Cd between the left $v s$ right hemispheres, with A10 (increased right Cd) vs A1 (increased left Cd) exposure.

\subsection{Brain responses to the ingredient $B$}


Brain activation maps (Figure 2A). Independent of the concentration used and similar to ingredient $\mathrm{A}$, ingredient $\mathrm{B}$ promoted brain responses in sensory brain regions related to olfaction and gustation, and in reward and motivational brain regions except for the Ac. A ten-fold increase of ingredient B concentration (B10 vs. B1) promoted an increased activation in the PP, in the anterior cingulate brain regions (Ventral-Anterior: VA-CC and DA-CC) and in prefrontal brain regions (Dorsal-Lateral Prefrontal Cortex: DL-PFC and A-PFC), except for the OFC. We could also detect a brain activity modulation in reward and motivational brain regions as well as in the associative learning brain regions except for the AMY.

Corrected SVC-based statistic (Figure 2B). We detected an impact of ingredient B1 toward an increased activation in the IC, the PHC, and toward a decreased activation in the $\mathrm{Cd}$ and the DL-PFC. A10 promoted similar modulations with additional features, such as an increased activation in the A-PFC, a tendency toward increased activation in the AMY and no modulation in the DL-PFC. A ten-fold increased of the ingredient B concentration (B10 vs B1) promoted a decreased activation in the IC, and a tendency toward increased activation in the A-PFC and the PUT.

\subsection{Different brain responses to the ingredient $A$ and $B$}

Brain activation maps (Figure 3A). A10 and B10 were able to promote different modulations of brain activity. A10 promoted higher brain responses in the $\mathrm{Cd}$, a reward and motivational brain region, in the frontal lobes, including OFC but not A-PFC, whereas B10 promoted higher brain activation in the A-PFC, and in cingulate brain regions (DP-CC, VA-CC, DA$\mathrm{CC}$ ), and in associative learning brain regions, including the AMY and the PHC.

Corrected SVC-based statistic (Figure 3B). With respect to the brain activation maps, A10 was able to promote a higher activation in the reward and motivational brain region $(\mathrm{Cd})$ whereas B10 promoted a higher activation in the A-PFC. 


\section{Discussion}

For the first time, we described in the pig model the fMRI BOLD brain responses to contrasted and novel olfactory stimulations. We were able to detect the brain responses elicited by two plant-based sensory functional feed ingredients at two different concentrations. We also provided a comparison of the brain responses to these two ingredients at the higher concentration, for which higher activation of the reward and cognitive areas were detected compared to the lower concentrations tested.

A previous study from our lab had investigated, with fluoro-deoxy-glucose (FDG) positron emission tomography (PET), the brain responses under dual gustatory and olfactory stimulation from a single food ingredient with or without previous exposure, i.e. familiarization from weaning to testing a month later (Val-Laillet, Meurice, \& Clouard, 2016). The ingredient used for this previous brain imaging study (similar to ingredient $\mathrm{A}$ in the present study) was chosen on the basis of the behavioral responses elicited by different feed ingredients at different concentrations (Clouard \& Val-Laillet, 2014), because it led to increased palatability and acceptance of an unfamiliar diet the day of feed transition. Here, we implemented the experimental stimulation set-up to fMRI in order to investigate, within the same imaging session and without prior familiarization to the ingredients, the brain responses under olfactory stimulation with two different feed ingredients at two different concentrations. We have previously shown the potential of fMRI in the pig model for the screening of brain responses to pleasant/unpleasant gustatory stimulations (Coquery et al., 2018). In the present study, our paradigm was successful in screening the brain responses to each ingredient and allowed for direct comparison between them.

The main limitation of our paradigm is that the ingredient $\mathrm{B}$ was always tested after the ingredient $\mathrm{A}$, which implies a possible order bias in brain activation. Interpretation of the 
comparison between ingredients $\mathrm{A}$ and $\mathrm{B}$ must be done with cautious then, since brain responses might have been different had the order of exposure to the two ingredients been reversed. It is reassuring though to note that no general increase or decrease in brain activity was observed along time during the fMRI session, since the comparison between ingredients $\mathrm{A}$ and $\mathrm{B}$ led to increased responses in some brain areas and decreased responses in others. Be that as it may, for future experiments, the presentation order should be reversed in half of the subjects to compensate for this hypothetical order bias.

The choice of odor concentration is a major issue considering that, depending on the concentration used, a given olfactory compound might yield contrasted or even opposite responses, from pleasantness to aversion (Clouard, Jouhanneau, Meunier-Salaün, Malbert, \& Val-Laillet, 2012). Here, a ten-fold increase of ingredients A and B promoted an increased BOLD signal in the PP, a primary olfactory center/relay, and in frontal brain areas, suggesting a potential increased perception and integration along with increased concentration. Interestingly, a decreased BOLD signal was detected in the IC (gustatory and associative cortex), which usually also responds to olfactory stimulation, especially for taste-like aspects of food odors (Veldhuizen, Nachtigal, Teulings, Gitelman, \& Small, 2010). Here, we used the orthonasal route for stimulation, which has been shown to promote distinct brain responses compared to the retronasal route (Small, Gerber, Mak, \& Hummel, 2005). Additionally, the animals were naïve to both ingredients, preventing any association between these ingredients and the feed. This might account for a surprisingly decreased response in the IC with increased ingredient concentration. A ten-fold increase of ingredient A concentration (A10 vs. A1) tended to induce higher activation in the reward and motivational areas, i.e. the $\mathrm{Cd}$ and the PUT, but also, as seen with the global activation maps, to promote increased activation in the Ac which is involved in reward, and in the OFC, a brain region involved in olfactory/gustatory food valence (Anderson et al., 2003) and pleasantness/hedonism 
(Grabenhorst, Rolls, Margot, Silva, \& Velazco, 2007; Zou, van Hartevelt, Kringelbach, Cheung, \& Chan, 2016)11/26/2019 9:39:00 AM. Overall these observations suggested an increased pleasantness specific to the increase in ingredient A concentration.

In our study, both sensory ingredients A and B promoted brain responses in brain structures responding during early stages of odor processing such as the primary olfactory cortex (PP), hippocampus (HPC), PHC, AMY, and OFC (Stadlbauer et al., 2016). However, compared to each other, and at the higher concentration used, the two ingredients promoted contrasting brain responses. Ingredient $\mathrm{A}$ is mainly composed of Citrus sinensis, a food extract from sweet orange that has been reported to promote a broad spectrum of effects (Dosoky \& Setzer, 2018) and was already tested in the pig using PET neuroimaging (Val-Laillet, Meurice, \& Clouard, 2016), whereas ingredient B contained Origanum vulgarae, of which one of the main active compounds is carvacrol, a molecule promoting positive effects in human health (Sharifi-Rad et al., 2018), but also modulating behavior in lambs (Simitzis, Deligeorgis, Bizelis, \& Fegeros, 2008) and depression-like behavior in rat (Amiresmaeili et al., 2018). Ingredient B also contained Cymbopogon flexuosus, the so-called lemongrass, which has been reported in mice to elicit positive effects on mood regulation (Blanco et al., 2009), and notably reduced anxiety (Costa et al., 2011). Previous studies in humans either showed a reduced activation in the prefrontal cortex in association with "comfort" feeling after orange oil inhalation (Igarashi et al., 2014), or an increased cerebral blood flow in the prefrontal cortex associated with increased cognitive functions in response to flavone-rich citrus juice (Lamport et al., 2016). In our study, exposure to ingredient A promoted a lower activation in the A-PFC compared to exposure to ingredient $\mathrm{B}$, but a higher activation in the DL-PFC, as seen in the global activation maps. The anterior (memory and perception) and dorsolateral (executive functions and attention) parts of the prefrontal cortex are linked to different cognitive processes, which probably explains the different outcomes observed in our study. 
We showed here that the two ingredients used modulated differently both these areas via olfaction only. It would be very interesting to assess their effects in a pig model of chronic psychosocial stress and/or anxio-depressive symptoms for example, as modulations of the aforementioned brain areas were recently described in this model (Menneson et al. 2019). Ingredient A promoted a higher activation in the reward/motivation brain regions, especially the $\mathrm{Cd}$, which is in line with the activation in dopaminergic brain areas with food-related odors in humans (Sorokowska et al., 2017). It is also interesting to notice that the higher concentration of ingredient $\mathrm{A}$ activated the right $\mathrm{Cd}$ compared to its lower concentration, and that a reversed effect was observed in the left $\mathrm{Cd}$. Despite that carvacrol has also been reported to promote similar effects on the dopaminergic system in rat (Zotti et al., 2013), and to enhance well-being in humans (Mechan et al., 2011), the activation in the related-brain regions, i.e. dorsal striatum (Cd and PUT) and OFC, was lower with ingredient $\mathrm{B}$. This suggested that, at the concentrations used, ingredient A promoted higher pleasantness than ingredient $\mathrm{B}$, which needs to be further confirmed by behavioral tests. Additional brain exploratory methods, such as in vivo nuclear brain imaging (Gautier et al., 2018) or postmortem brain immunohistochemistry, might also be used to confirm that the dopaminergic system is specifically involved in the brain response to feed ingredients, with a focus on the striatum and prefrontal cortex, which are well-known dopaminergic areas that significantly responded to the olfactory stimulations in our study.

Exposure to ingredient B promoted higher brain responses in the AMY compared to exposure to ingredient A, as seen with the global activation maps. In humans, AMY is involved in olfactory hedonic processing-related tasks (Zou et al., 2016), and the higher activation with ingredient B could suggest a higher odor emotional salience (Winston, Gottfried, Kilner, \& Dolan, 2005). However, given that carvacrol promotes in humans a perception of "warmth" through trigeminal stimulation (Klein et al., 2014), and that the AMY has been shown in rats 
to receive trigeminal inputs (Hummel \& Livermore, 2002), the higher activation in the AMY obtained with ingredient $\mathrm{B}$ compared to A might also be a consequence of the trigeminal stimulation. This is in line with a recent human study (Fournel, Ferdenzi, Sezille, Rouby, \& Bensafi, 2016).

Finally, the olfactory stimulations used in our imaging paradigm did not permit to detect any modulation of brain activity in homeostatic brain areas, such as the hypothalamus. First, this might be due to limitations of the imaging method because the hypothalamus is a small brain structure composed of several specific nuclei, which complicates acquisition of images with a good resolution. The hypothalamus is also endowed with fenestrated capillaries probably enhancing interface imaging artifacts or noise. Second, we might also hypothesize that olfactory stimulation only was not sufficient in our study to modulate homeostatic signals and the hunger/satiety internal state, and that the observed effects were restricted to the hedonic dimension of eating control. Physiological measurements (e.g. gut hormones) concomitant to the olfactory stimulation might help in the future to disentangle this question.

\section{Conclusion}

We provided here a global comparison of the brain responses to two sensory functional feed ingredients at two different concentrations with fMRI in the pig model, and showed that increased concentration of ingredients promoted different brain responses, which were also dependent on the ingredient composition. The ingredient A, mainly composed of Citrus sinensis extracts was able to elicit higher brain responses in brain areas involved in pleasantness, compared to ingredient B that was principally composed of Origanum vulgarae and Cymbopogon flexuosus extracts. Our findings might find relevance in the scope of the growing interest in the understanding of emotion regulation through olfaction modulation (Billot et al., 2017; Soudry et al., 2011). 


\section{Acknowledgements}

This study and the PhD grant of Sophie Menneson were funded by Phodé, the ANRT (Agence Nationale Recherche Technologie, French Association for Research and Technology), and INRA. We thank the staff from the animal facility UEPR (Unité Expérimentale Porcs de Rennes, St Gilles, France) of INRA for their technical support: Serge Dubois, Bruno Fontaine, Renan Delaunay, Alain Chauvin. We acknowledge the PRISM (Plateforme de Recherche en Imagerie et Spectroscopie Multimodales, Rennes, France) core facility for its technical support, and especially Stéphane Quellec.

\section{Conflict of interest statement}

The functional feed/food ingredients used in this study were designed by Phodé that also cofunded, together with the ANRT (French Association for Research and Technology) and INRA, the research project and $\mathrm{PhD}$ grant of Sophie MENNESON. Authors Sophie MENNESON, Virginie NOIROT, and Pierre ETIENNE were employed by company Phodé. All other authors declare no competing interests.

\section{Authors' contribution}

Experimental design: NC, SM, PE, VN, and DVL. Technical development: NC, RJ, and PM. Performing the experiments: NC, SM, PM, and RJ. Data analysis: NC. Manuscript writing: NC and DVL. Manuscript revising: all co-authors. 


\section{References}

Amiresmaeili, A., Roohollahi, S., Mostafavi, A., \& Askari, N. (2018). Effects of oregano essential oil on brain TLR4 and TLR2 gene expression and depressive-like behavior in a rat model. Research in Pharmaceutical Sciences, 13(2), 130-141. https://doi.org/10.4103/17355362.223795

Anderson, A. K., Christoff, K., Stappen, I., Panitz, D., Ghahremani, D. G., Glover, G., ... Sobel, N. (2003). Dissociated neural representations of intensity and valence in human olfaction. Nature Neuroscience, 6(2), 196-202. https://doi.org/10.1038/nn1001

Billot, P.-E., Andrieu, P., Biondi, A., Vieillard, S., Moulin, T., \& Millot, J.-L. (2017).

Cerebral bases of emotion regulation toward odours: A first approach. Behavioural Brain Research, 317, 37-45. https://doi.org/10.1016/j.bbr.2016.09.027

Blanco, M. M., Costa, C. A. R. A., Freire, A. O., Santos, J. G., \& Costa, M. (2009). Neurobehavioral effect of essential oil of Cymbopogon citratus in mice. Phytomedicine, 16(2), 265-270. https://doi.org/10.1016/j.phymed.2007.04.007

Bower, A. M., Real Hernandez, L. M., Berhow, M. A., \& de Mejia, E. G. (2014). Bioactive Compounds from Culinary Herbs Inhibit a Molecular Target for Type 2 Diabetes Management, Dipeptidyl Peptidase IV. Journal of Agricultural and Food Chemistry, 62(26), 6147-6158. https://doi.org/10.1021/jf500639f

Cairo, P. L. G., Gois, F. D., Sbardella, M., Silveira, H., de Oliveira, R. M., Allaman, I. B., ... Costa, L. B. (2018). Effects of dietary supplementation of red pepper (Schinus terebinthifolius Raddi) essential oil on performance, small intestinal morphology and microbial counts of weanling pigs. Journal of the Science of Food and Agriculture, 98(2), 541-548. https://doi.org/10.1002/jsfa.8494

Clouard, C., Jouhanneau, M., Meunier-Salaün, M.-C., Malbert, C.-H., \& Val-Laillet, D. (2012). Exposures to Conditioned Flavours with Different Hedonic Values Induce Contrasted 
Behavioural and Brain Responses in Pigs. PLOS ONE, 7(5), e37968.

https://doi.org/10.1371/journal.pone.0037968

Clouard, C., \& Val-Laillet, D. (2014). Impact of sensory feed additives on feed intake, feed preferences, and growth of female piglets during the early postweaning period. Journal of Animal Science, 92(5), 2133-2140. https://doi.org/10.2527/jas.2013-6809

Coquery, N., Meurice, P., Janvier, R., Bobillier, E., Quellec, S., Fu, M., ... Val-Laillet, D. (2018). fMRI-based brain responses to quinine and sucrose gustatory stimulation for nutrition research in the minipig model: a proof of concept study. Frontiers in Behavioral Neuroscience, 12. https://doi.org/10.3389/fnbeh.2018.00151

Costa, C. A. R. de A., Kohn, D. O., de Lima, V. M., Gargano, A. C., Flório, J. C., \& Costa, M. (2011). The GABAergic system contributes to the anxiolytic-like effect of essential oil from Cymbopogon citratus (lemongrass). Journal of Ethnopharmacology, 137(1), 828-836. https://doi.org/10.1016/j.jep.2011.07.003

Dosoky, N. S., \& Setzer, W. N. (2018). Biological Activities and Safety of Citrus spp. Essential Oils. International Journal of Molecular Sciences, 19(7), 1966. https://doi.org/10.3390/ijms19071966

Dudek-Wicher, R. K., Junka, A., \& Bartoszewicz, M. (2018). The influence of antibiotics and dietary components on gut microbiota. Przeglad Gastroenterologiczny, 13(2), 85-92. https://doi.org/10.5114/pg.2018.76005

Fournel, A., Ferdenzi, C., Sezille, C., Rouby, C., \& Bensafi, M. (2016). Multidimensional representation of odors in the human olfactory cortex. Human Brain Mapping, 37(6), 21612172. https://doi.org/10.1002/hbm.23164

Gautier, Y., Luneau, I., Coquery, N., Meurice, P., Malbert, C.-H., Guerin, S., ... Val-Laillet, D. (2018). Maternal Western diet during gestation and lactation modifies adult offspring's cognitive and hedonic brain processes, behavior, and metabolism in Yucatan minipigs. The 
FASEB Journal, fj.201701541. https://doi.org/10.1096/fj.201701541

Grabenhorst, F., Rolls, E. T., Margot, C., Silva, M. A. A. P. da, \& Velazco, M. I. (2007). How Pleasant and Unpleasant Stimuli Combine in Different Brain Regions: Odor Mixtures.

Journal of Neuroscience, 27(49), 13532-13540. https://doi.org/10.1523/JNEUROSCI.3337-

07.2007

Hummel, T., \& Livermore, A. (2002). Intranasal chemosensory function of the trigeminal nerve and aspects of its relation to olfaction. International Archives of Occupational and Environmental Health, 75(5), 305-313. https://doi.org/10.1007/s00420-002-0315-7

Igarashi, M., Ikei, H., Song, C., \& Miyazaki, Y. (2014). Effects of olfactory stimulation with rose and orange oil on prefrontal cortex activity. Complementary Therapies in Medicine, 22(6), 1027-1031. https://doi.org/10.1016/j.ctim.2014.09.003

Jugl-Chizzola, M., Ungerhofer, E., Gabler, C., Hagmüller, W., Chizzola, R., Zitterl-Eglseer, K., \& Franz, C. (2006). Testing of the palatability of Thymus vulgaris L. and Origanum vulgare L. as flavouring feed additive for weaner pigs on the basis of a choice experiment. Berliner Und Munchener Tierarztliche Wochenschrift, 119(5-6), 238-243.

Klein, A. H., Joe, C. L., Davoodi, A., Takechi, K., Carstens, M. I., \& Carstens, E. (2014). Eugenol and carvacrol excite first- and second-order trigeminal neurons and enhance their heat-evoked responses. Neuroscience, 271, 45-55.

https://doi.org/10.1016/j.neuroscience.2014.04.019

Lamport, D. J., Pal, D., Macready, A. L., Barbosa-Boucas, S., Fletcher, J. M., Williams, C. M., ... Butler, L. T. (2016). The effects of flavanone-rich citrus juice on cognitive function and cerebral blood flow: an acute, randomised, placebo-controlled cross-over trial in healthy, young adults. British Journal of Nutrition, 116(12), 2160-2168.

https://doi.org/10.1017/S000711451600430X

Mechan, A. O., Fowler, A., Seifert, N., Rieger, H., Wöhrle, T., Etheve, S., ... Mohajeri, M. H. 
(2011). Monoamine reuptake inhibition and mood-enhancing potential of a specified oregano extract. British Journal of Nutrition, 105(8), 1150-1163.

https://doi.org/10.1017/S0007114510004940

Menneson, S., Ménicot, S., Ferret-Bernard, S., Guérin, S., Romé, V., Le Normand, L.,

Randuineau, G., Gambarota, G., Noirot, V., Etienne, P., Coquery, N., Val-Laillet, D. 2019.

Validation of a psychosocial chronic stress model in the pig using a multidisciplinary

approach at the gut-brain and behavior levels. Frontiers in Behavioral Neuroscience, in press. https://doi: 10.3389/fnbeh.2019.00161

Patra, A. K., Amasheh, S., \& Aschenbach, J. R. (2018). Modulation of gastrointestinal barrier and nutrient transport function in farm animals by natural plant bioactive compounds $-\mathrm{A}$ comprehensive review. Critical Reviews in Food Science and Nutrition, 0(0), 1-30.

https://doi.org/10.1080/10408398.2018.1486284

Piątkowska, E., \& Rusiecka-Ziółkowska, J. (2016). Influence of Essential Oils on Infectious Agents. Advances in Clinical and Experimental Medicine: Official Organ Wroclaw Medical University, 25(5), 989-995.

Pires, L. F., Costa, L. M., Silva, O. A., de Almeida, A. A. C., Cerqueira, G. S., de Sousa, D. P., \& de Freitas, R. M. (2013). Anxiolytic-like effects of carvacryl acetate, a derivative of carvacrol, in mice. Pharmacology Biochemistry and Behavior, 112, 42-48.

https://doi.org/10.1016/j.pbb.2013.09.001

Saikali, S., Meurice, P., Sauleau, P., Eliat, P.-A., Bellaud, P., Randuineau, G., ... Malbert, C.H. (2010). A three-dimensional digital segmented and deformable brain atlas of the domestic pig. Journal of Neuroscience Methods, 192(1), 102-109. https://doi.org/10.1016/j.jneumeth.2010.07.041

Sharifi-Rad, M., Varoni, E. M., Iriti, M., Martorell, M., Setzer, W. N., Del Mar Contreras, M., ... Sharifi-Rad, J. (2018). Carvacrol and human health: A comprehensive review. 
Phytotherapy Research: PTR. https://doi.org/10.1002/ptr.6103

Simitzis, P. E., Deligeorgis, S. G., Bizelis, J. A., \& Fegeros, K. (2008). Feeding preferences in lambs influenced by prenatal flavour exposure. Physiology \& Behavior, 93(3), 529-536. https://doi.org/10.1016/j.physbeh.2007.10.013

Small, D. M., Gerber, J. C., Mak, Y. E., \& Hummel, T. (2005). Differential Neural Responses Evoked by Orthonasal versus Retronasal Odorant Perception in Humans. Neuron, 47(4), 593605. https://doi.org/10.1016/j.neuron.2005.07.022

Sorokowska, A., Schoen, K., Hummel, C., Han, P., Warr, J., \& Hummel, T. (2017). FoodRelated Odors Activate Dopaminergic Brain Areas. Frontiers in Human Neuroscience, 11. https://doi.org/10.3389/fnhum.2017.00625

Soudry, Y., Lemogne, C., Malinvaud, D., Consoli, S.-M., \& Bonfils, P. (2011). Olfactory system and emotion: Common substrates. European Annals of Otorhinolaryngology, Head and Neck Diseases, 128(1), 18-23. https://doi.org/10.1016/j.anorl.2010.09.007

Stadlbauer, A., Kaltenhäuser, M., Buchfelder, M., Brandner, S., Neuhuber, W. L., \& Renner, B. (2016). Spatiotemporal Pattern of Human Cortical and Subcortical Activity during EarlyStage Odor Processing. Chemical Senses. https://doi.org/10.1093/chemse/bjw074

Val-Laillet, D., Meurice, P., \& Clouard, C. (2016). Familiarity to a Feed Additive Modulates Its Effects on Brain Responses in Reward and Memory Regions in the Pig Model. PLOS ONE, 11(9), e0162660. https://doi.org/10.1371/journal.pone.0162660 van Breda, S. G. J., \& de Kok, T. M. C. M. (2018). Smart Combinations of Bioactive Compounds in Fruits and Vegetables May Guide New Strategies for Personalized Prevention of Chronic Diseases. Molecular Nutrition \& Food Research, 62(1).

https://doi.org/10.1002/mnfr.201700597

Veldhuizen, M. G., Nachtigal, D., Teulings, L., Gitelman, D. R., \& Small, D. M. (2010). The Insular Taste Cortex Contributes to Odor Quality Coding. Frontiers in Human Neuroscience, 
4. https://doi.org/10.3389/fnhum.2010.00058

Williams, A. R., Hansen, T. V. A., Krych, L., Ahmad, H. F. B., Nielsen, D. S., Skovgaard, K., \& Thamsborg, S. M. (2017). Dietary cinnamaldehyde enhances acquisition of specific antibodies following helminth infection in pigs. Veterinary Immunology and Immunopathology, 189, 43-52. https://doi.org/10.1016/j.vetimm.2017.06.004

Winston, J. S., Gottfried, J. A., Kilner, J. M., \& Dolan, R. J. (2005). Integrated Neural Representations of Odor Intensity and Affective Valence in Human Amygdala. Journal of Neuroscience, 25(39), 8903-8907. https://doi.org/10.1523/JNEUROSCI.1569-05.2005

Zotti, M., Colaianna, M., Morgese, M. G., Tucci, P., Schiavone, S., Avato, P., \& Trabace, L. (2013). Carvacrol: From Ancient Flavoring to Neuromodulatory Agent. Molecules, 18(6), 6161-6172. https://doi.org/10.3390/molecules18066161

Zou, L., van Hartevelt, T. J., Kringelbach, M. L., Cheung, E. F. C., \& Chan, R. C. K. (2016). The neural mechanism of hedonic processing and judgment of pleasant odors: An activation likelihood estimation meta-analysis. Neuropsychology, 30(8), 970-979.

https://doi.org/10.1037/neu0000292 


\section{Figure}

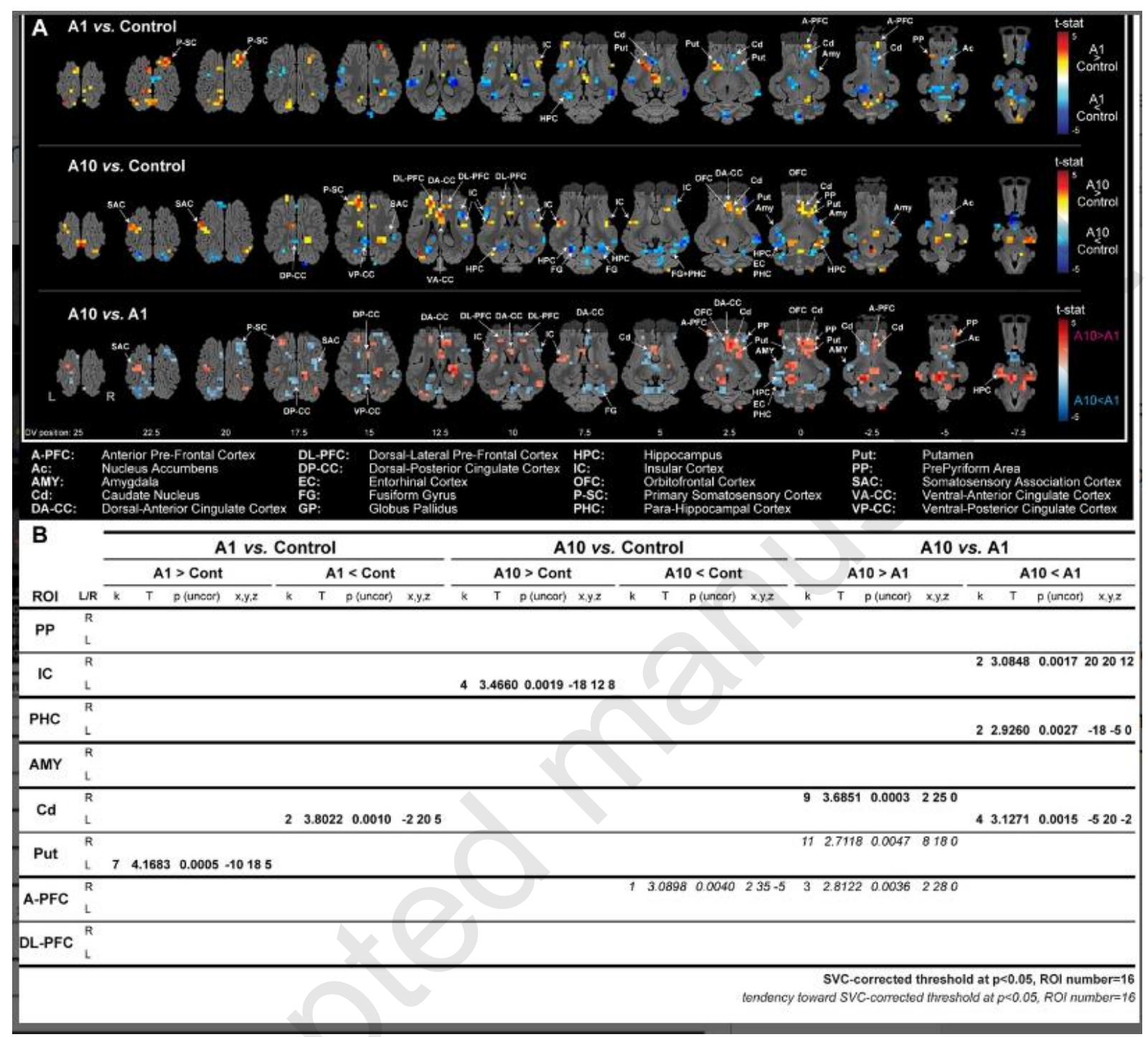

Figure 1-(A) Horizontal maps of global brain BOLD responses (contrast between average brain responses) to the functional ingredient A (at two concentrations: A1 and A10, which was ten-fold increased compared to A1) compared to control stimulations, and between the two concentrations of the ingredient A (A10 vs. A1). P-value threshold=0.05, k>4, DV: dorsal-ventral position in mm related to the posterior commissure. The part of the brain that was not covered with the average BOLD-based statistical maps is superimposed in dark grey on the anatomical maps (frontal cortices). (B) SVC-based statistics: related ROIs with uncorrected $p$-value that reach the criteria of $\mathrm{p}<0.05$ after Bonferroni correction for multiple 
ROIs comparison for sixteen regions of interest (ROI) from Saikali's atlas (Saikali et al., 2010) chosen upon a priori hypothesis. Statistical differences are depicted in bold, and tendency toward statistical differences are depicted in italic. L: ROI in the left hemisphere, and R: ROI in the right hemisphere. ROI abbreviations are detailed at the bottom of the panel A. 


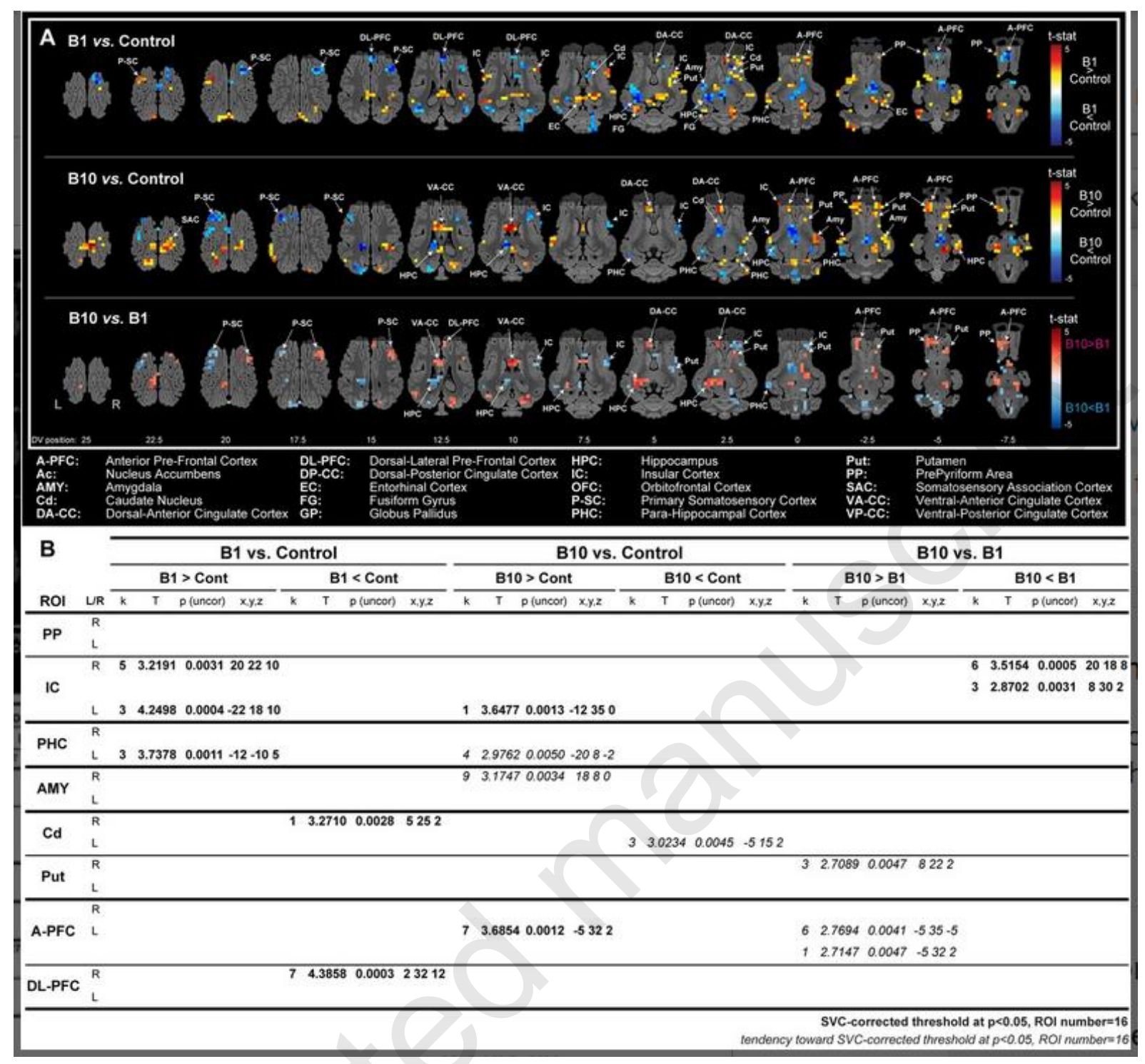

Figure 2-(A) Horizontal maps of global brain BOLD responses (contrast between average brain responses) to the ingredient B (at two concentrations: B1 and B10, which was ten-fold increased compared to B1) compared to control stimulations, and between the two concentrations of the ingredient B (B10 vs. B1). P-value threshold=0.05, k>4, DV: dorsalventral position in mm related to the posterior commissure. The part of the brain that was not covered with the average BOLD-based statistical maps is superimposed in dark grey on the anatomical maps (frontal cortices). (B) SVC-based statistics: related ROIs with uncorrected pvalue that reach the criteria of $\mathrm{p}<0.05$ after Bonferroni correction for multiple ROIs comparison for sixteen regions of interest (ROI) from Saikali's atlas (Saikali et al., 2010) 
chosen upon a priori hypothesis. Statistical differences are depicted in bold, and tendency toward statistical differences are depicted in italic. L: ROI in the left hemisphere, and R: ROI in the right hemisphere. ROI abbreviations are detailed at the bottom of the panel A. 


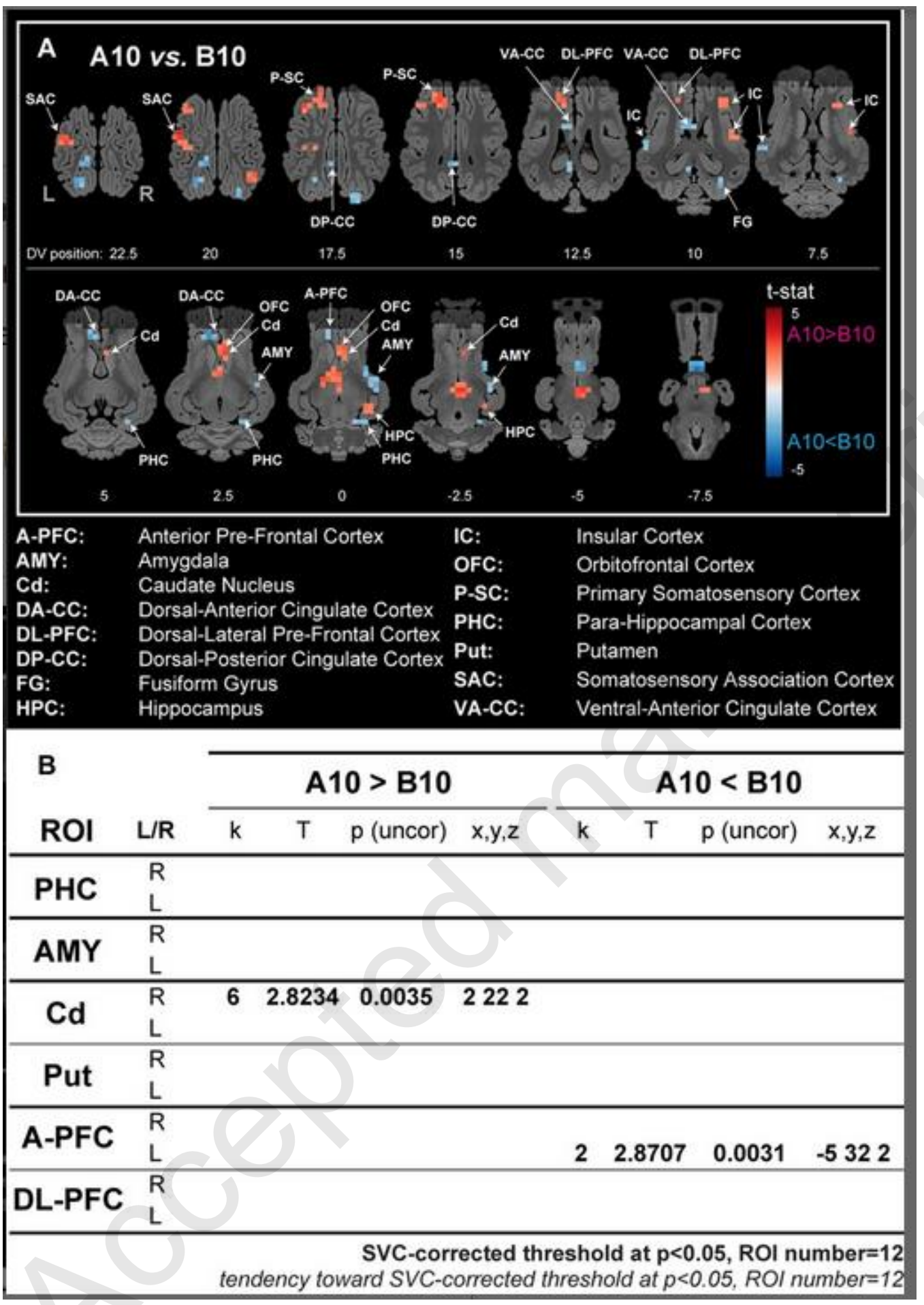

Figure 3-(A) Horizontal maps of global brain responses (contrast between average brain responses) between the ingredients A and B stimulations at the higher concentrations (A10 vs. B10). P-value threshold=0.05, DV: dorsal-ventral position in $\mathrm{mm}$ related to the posterior commissure. P-value threshold=0.05, $\mathrm{k}>4$, DV: dorsal-ventral position in $\mathrm{mm}$ related to the posterior commissure. The part of the brain that was not covered with the average BOLD- 
based statistical maps is superimposed in dark grey on the anatomical maps (frontal cortices). (B) SVC-based statistics: related ROIs with uncorrected p-value that reach the criteria of $\mathrm{p}<0.05$ after Bonferroni correction for multiple ROIs comparison for twelve regions of interest (ROI) from Saikali's atlas (Saikali et al., 2010) chosen upon a priori hypothesis. Statistical differences are depicted in bold, and tendency toward statistical differences are depicted in italic. L: ROI in the left hemisphere, and R: ROI in the right hemisphere. ROI abbreviations are detailed at the bottom of the panel A. 